\title{
Stimulus, task, and environmental characteristics as factors in the cognitive processing of English sentences
}

\author{
NANCY KALISH \\ California State University, Sacramento, California 95819 \\ and

\section{P. BRUCE LANDON, DARYLYNN S. RANK, and PETER SUEDFELD University of British Columbia, Vancouver, British Columbia V6T IW5, Canada}

\begin{abstract}
The cognitive processing of sentences as a function of stimulus and environmental factors was examined. Sentences were varied as to the number of modifiers per noun (difficulty) and self-embedded phrases (complexity). The experimental environment was $24 \mathrm{~h}$ of restricted stimulation, which was compared with a nondeprived control environment. The dependent measures were recall and paraphrasing. Increasing difficulty and complexity independently led to decrements on both types of performance; an interaction effect showed that difficulty had a more powerful effect on recall, with a trend in the same direction for paraphrasing. Stimulus restriction heightened the negative effects of increasing complexity and difficulty on paraphrasing, but it had no such effect on recall.
\end{abstract}

The global environment in which information processing takes place has been largely ignored by cognitive psychologists. However, researchers have been interested in the relationship between performance and levels of stimulus input. For example, the effects of the restricted environmental stimulation technique (REST) interact with task characteristics, a finding that has implications for cognitive theories of arousal and level of processing (e.g., Craik \& Tulving, 1975; Taylor \& Spence, 1952), as well as for the combination of REST with verbal messages in habit modification, language learning, and other applied contexts (Suedfeld, 1980).

This study investigates the roles of REST and two stimulus variables in the recall and comprehension of English sentences. The stimulus variables are (1) difficulty, closely related to memory load and defined by such factors as the number and accessibility of discriminable items of information that must be processed, and (2) complexity, the degree of active cognitive manipulation required or permitted by the problem (Suedfeld \& Landon, 1970). For example, the difficulty of digit recall depends on the number of digits; with the addition of transformations, such as reversal, complexity

This work was supported by a grant from the Natural Sciences and Engineering Research Council of Canada to P. Suedfeld. Carmenza Ramirez and Thomas Maxfield provided valuable assistance. The order of authors is alphabetical; requests for reprints should be addressed to Peter Suedfeld, Department of Psychology, University of British Columbia, Vancouver, British Columbia V6T 1W5, Canada. increases. The experiment also included relatively simple and complex performance measures (recall and paraphrase, respectively).

We varied the number of modifiers per noun (difficulty) and the number of self-embedded clauses (complexity) in the sentences. Each sentence included two concepts. In regular constructions, ideas occur sequentially; in self-embedded sentences, the subject and verb of a clause are separated by another clause. The expression thus becomes more complex; compare "The dog bit the child who liked the man that ran away" (regular) and "The man whom the child that the dog bit liked ran away" (self-embedded). Self-embeddedness has previously been characterized as a complexity factor (Fodor \& Garrett, 1967), although some authors use complexity and difficulty interchangeably (e.g., McDaniel, 1981). McDaniel found that self-embedded sentences were more difficult to understand, but not to remember, than their regular equivalents.

We measured the accuracy of retention and of paraphrasing, the latter requiring active cognitive manipulation. The purpose was to see whether common variables affect both measures and whether the influence of the two stimulus dimensions on the two types of tasks would be similar.

\section{METHOD}

\section{Subjects}

Twenty volunteers who answered advertisements offering $\$ 20$ to stay $24 \mathrm{~h}$ in a laboratory were randomly assigned to either the REST or the control group. 


\section{Stimuli and Measures}

Forty-five sentences were constructed. Difficulty was manipulated by having zero, one, or two adjectives for each noun; complexity was operationalized as the number (zero, one, or two) of self-embedded clauses within the sentence. The task was to repeat each sentence verbatim and then to paraphrase it. Each of the five stimulus sets consisted of nine tape-recorded sentences constructed according to the 3 by 3 matrix described above. The tape was stopped after each sentence until the subject finished answering. To keep the modifiers equivalent, each contained two syllables and one morpheme. To keep meanings equivalent, each sentence contained three subject constituents.

Recall errors were misplacements, additions, substitutions, and omissions of words. Paraphrase scores (0-3) were the number of clauses that the subject failed to explain correctly.

\section{Procedure}

Subjects did the tasks once 2 days before the experiment. REST subjects spent the experimental day on a bed in a soundproof dark chamber (see Suedfeld, 1980, for procedural details). Control subjects spent the $24 \mathrm{~h}$ in a normal room adjoining the chamber.

Both groups were told that they would hear sentences similar to the familiarization set. Tests occurred as soon as the subject was settled in $(0 \mathrm{~h})$, after $12 \mathrm{~h}$, and, last, at $24 \mathrm{~h}$. The order of the sentence sets was randomized across subjects.

\section{RESULTS}

Paraphrase errors were moderately correlated with total recall errors $(\mathrm{r}=.57, \mathrm{p}<.01)$, which was expected, since the subject who failed to remember a sentence correctly would be likely to make mistakes in paraphrasing it. Table 1 shows the mean scores.

There was no overall difference on either measure as a function of REST, and there were no interactions on recall. However, the REST by Difficulty and REST by Complexity interactions were significant on paraphrasing $[F(2,36)=37.28$ and 13.59 , both $\mathrm{ps}<.001]$, with REST increasing the number of errors that occurred as a function of increasing complexity or difficulty. The triple interaction was also significant $[\mathrm{F}(4,72)=47.39$, $\mathrm{p}<.001]$. The REST group did worst in paraphrasing when both difficulty and complexity were high, and REST interfered with performance throughout the range of sentences.

Practice led to fewer recall errors $[\mathrm{F}(2,36)=7.0$, $\mathrm{p}<.005]$ (means $=5.6,4.6$, and 4.6 at 0,12 , and $24 \mathrm{~h}$ ). No interactive effect of increasing REST duration and no practice effect on paraphrasing were found.

Increasing difficulty led to more errors in recall $[\mathrm{F}(2,36)=285.31, \mathrm{p}<.0001]$ and in paraphrasing $[F(2,36)=18.61, p<.001]$. All of the paired comparisons showed significant differences. Increasing complexity also led to more errors in recall $[\mathrm{F}(2,36)=16.71$, $\mathrm{p}<.001]$ and in paraphrasing $[\mathrm{F}(2,36)=5.79, \mathrm{p}<.01]$. The difference between the high-complexity condition and the other two was significant by Newman-Keuls rests on both measures.

The Difficulty by Complexity interaction was significant for recall $[\mathrm{F}(4,72)=5.48, \mathrm{p}<.001]$ and almost
Table 1

Mean Scores as a Function of Difficulty and Complexity

\begin{tabular}{lcccc}
\hline & \multicolumn{4}{c}{ Difficulty } \\
\cline { 2 - 5 } Complexity & Low & Medium & High & Mean \\
\hline & \multicolumn{4}{c}{ Recall Errors } \\
Low & .63 & 3.48 & 8.78 & 4.30 \\
Medium & 1.06 & 3.70 & 7.80 & 4.19 \\
High & 1.73 & 6.73 & 9.66 & 6.04 \\
Mean & 1.14 & 4.64 & 8.75 & 4.84 \\
& & Paraphrase & Accuracy \\
Low & 2.78 & 2.36 & 2.12 & 2.42 \\
Medium & 2.60 & 2.48 & 2.30 & 2.46 \\
High & 2.52 & 2.33 & 1.70 & 2.18 \\
Mean & 2.63 & 2.39 & 2.04 & 2.35 \\
\hline
\end{tabular}

significant for paraphrase $[F(4,72)=2.19, p=.07]$. Paired comparisons showed that performance was worst on high-complexity and high-difficulty sentences. Difficulty appeared to be the more powerful: High difficulty led to significantly poorer performance regardless of complexity level (all ps<.01). Within each difficulty level, increasing complexity was systematically associated with performance decrements.

Of the 1,432 recall errors, $587(41 \%)$ involved the modifiers used to manipulate the difficulty of the sentences. There were errors on $54 \%$ of the 1,080 adjectives. Of the 320 substitution errors, 112 (35\%) preserved the meaning (e.g., lady to woman, sofa to couch, aging to old, etc.).

\section{DISCUSSION}

The simultaneous variation of both stimulus and task characteristics related differentially to levels of processing was an important aspect of this study. The findings confirmed that self-embedded clauses can be used to manipulate levels of processing complexity and supported the argument that complexity and difficulty can be manipulated independently.

The findings were consistent with previous REST research. The impact of restriction was most evident on the complex response (paraphrase), which consistently deteriorated as a function of the requirement for deeper levels of processing across our two stimulus dimensions, whereas recall accuracy remained stable. This has implications for the identification of differential cognitive and neural effects of REST, and it may explain why concrete technical information concerning exercise and dieting adds significantly to the efficacy of REST, whereas more abstract persuasive messages related to smoking cessation have consistently failed to do so (Borrie \& Suedfeld, 1980; Suedfeld, 1980).

\section{REFERENCES}

Borrie, R. A., \& Suedfeld, P. Restricted environmental stimulation therapy in a weight reduction program. Journal of Behavioral Medicine, 1980, 3, 147-161.

Craik, F. I. M., \& Tulving, E. Depth of processing and the retention of words in episodic memory. Journal of Experimental Psychology: General, 1975, 104, 268-294.

Fodor, J. A., \& GARRET, M. Some syntactic determinants of 
sentential complexity. Perception \& Psychophysics, 1967, 2, 289-296.

McDaniel, M. A. Syntactic complexity and elaborative processing. Memory \& Cognition, 1981, 9, 487-495.

SuedFeld, P. Restricted environmental stimulation: Research and clinical applications. New York: Wiley, 1980.

Suedfeld, P., \& Landon, P. B. Motivational arousal and task complexity: Support for a model of cognitive changes in sensory deprivation. Journal of Experimental Psychology, 1970, 83, 329-330.

TAylor, J. A., \& Spence, K. W. The relationship of anxiety level to performance in serial learning. Journal of Experimental Psychology, 1952, 44, 61-64.

(Received for publication October 31, 1982.) 